\title{
CONVERGENCE OF NUMERICAL METHODS AND PARAMETER DEPENDENCE OF MIN-PLUS EIGENVALUE PROBLEMS, FRENKEL-KONTOROVA MODELS AND HOMOGENIZATION OF HAMILTON-JACOBI EQUATIONS
}

\author{
NICOLAS BACAËR ${ }^{1}$
}

\begin{abstract}
Using the min-plus version of the spectral radius formula, one proves: 1) that the unique eigenvalue of a min-plus eigenvalue problem depends continuously on parameters involved in the kernel defining the problem; 2) that the numerical method introduced by Chou and Griffiths to compute this eigenvalue converges. A toolbox recently developed at I.n.r.i.a. helps to illustrate these results. Frenkel-Kontorova models serve as example. The analogy with homogenization of Hamilton-Jacobi equations is emphasized.
\end{abstract}

Mathematics Subject Classification. 65J99, 65Z05.

Received: June 25, 2001. Revised: August 16, 2001.

\section{INTRODUCTION}

Some optimization problems can be reformulated using the semi-ring $\mathbb{R}_{\min }=(\mathbb{R} \cup\{+\infty\}, \oplus, \otimes)$ where

$$
\lambda \oplus \mu=\min (\lambda, \mu) \quad \text { and } \quad \lambda \otimes \mu=\lambda+\mu,
$$

so that they appear as analogues of classical linear eigenvalue problems. For example,

$$
\min _{1 \leq j \leq n}\left\{K_{i, j}+u_{j}\right\}=\lambda+u_{i} \text { and } \sum_{1 \leq j \leq n} K_{i, j} \times u_{j}=\lambda \times u_{i}
$$

look similar, and so do

$$
\min _{a \leq y \leq b}\{K(x, y)+u(y)\}=\lambda+u(x) \quad \text { and } \quad \int_{a}^{b} K(x, y) \times u(y) \mathrm{d} y=\lambda \times u(x) .
$$

These analogies have been used to develop over the semi-ring $\mathbb{R}_{\min }$ counterparts to the spectral theory of matrices [5] and of integral operators [14]. A numerical method for "min-plus integral eigenvalue problems" has been used in solid-state physics to draw phase diagrams of Frenkel-Kontorova models [6]. The main purpose of this paper is to prove the convergence of this method.

Keywords and phrases. Min-plus eigenvalue problems, numerical analysis, Frenkel-Kontorova model, Hamilton-Jacobi equations.

1 Université Paris 6, Laboratoire d'Analyse Numérique, 175 rue du chevaleret, 75013 Paris, France.

e-mail: bacaer@ann.jussieu.fr 
Section 1, inspired by [12], recalls how the analogies can be formalized by introducing some general definitions.

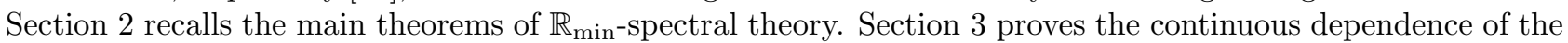
eigenvalue $\lambda$ on parameters possibly involved in the definition of the kernel $K$. Section 4 proves the convergence

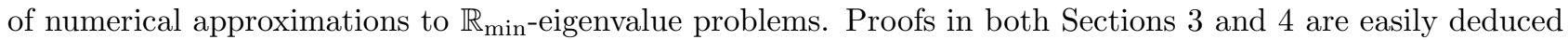
from a kind of spectral radius formula presented in Section 2. Section 5 is concerned with the case of periodic kernels. Section 6 illustrates the previous results in two contexts: Frenkel-Kontorova models in solid-state physics, and homogenization of Hamilton-Jacobi equations.

Let us mention that parameter dependent eigenvalue problems for Hamilton-Jacobi equations (which are equivalent to min-plus eigenvalue problems) also appear in the study of travelling fronts in solid propellant combustion $[3,15]$. This application was the initial motivation for the present study. But since the technical details are more complicated, they will be explained elsewhere.

Notice also that the numerical analysis of other min-plus linear problems is not always as straightforward as that of the eigenvalue problem presented here (see [4] for a discussion).

\section{Generalized Linear Algebra}

Definition 1.1. Let $\mathcal{R}$ be a set equipped with an operation + . One says that $(\mathcal{R},+)$ is a semi-group if + is associative and has a neutral element. One says that $(\mathcal{R},+)$ is a commutative semi-group if moreover + is commutative.

Definition 1.2. Let $\mathcal{R}$ be a set equipped with two operations + and $\times$. One says that $(\mathcal{R},+, \times)$ is a semi-ring if $(\mathcal{R},+)$ is a commutative semi-group whose neutral element is called $0,(\mathcal{R}, \times)$ is a semi-group whose neutral element is called $1, \times$ is distributive with respect to + , and $\forall \lambda \in \mathcal{R}, 0 \times \lambda=\lambda \times 0=0$.

Example 1.3. $(\mathbb{R},+, \times)$ and $\left(\mathbb{R}_{+},+, \times\right)$are semi-rings.

Example 1.4. Set $\mathbb{R}_{\min }=\mathbb{R} \cup\{+\infty\}$. Then $\left(\mathbb{R}_{\min }, \min ,+\right)$ is a semi-ring with neutral elements $+\infty$ and 0 .

Definition 1.5. Let $(\mathcal{R},+, \times)$ be a semi-ring and $(X,+)$ be a commutative semi-group. Suppose that for all $\lambda \in \mathcal{R}$ and $x \in X$, an element of $X$ called $\lambda \cdot x$ is given. One says that $(X,+, \cdot)$ is a semi-module over $(\mathcal{R},+, \times)$ if $\forall \lambda, \mu \in \mathcal{R}, \forall x, y \in X$,

$$
\begin{aligned}
& (\lambda+\mu) \cdot x=\lambda \cdot x+\mu \cdot x ; \quad(\lambda \times \mu) \cdot x=\lambda \cdot(\mu \cdot x) ; \\
& \lambda \cdot(x+y)=\lambda \cdot x+\lambda \cdot y ; \quad 1 \cdot x=x .
\end{aligned}
$$

If $Y \subset X$ and $Y$ is a semi-module over $\mathcal{R}$, one says that $Y$ is a sub-semi-module of $X$.

Example 1.6. Let $X$ be a set and $(\mathcal{R},+, \times)$ be a semi-ring. For all $f, g \in \mathcal{R}^{X}$ and for all $x \in X$, set

$$
(f+g)(x)=f(x)+g(x), \quad(\lambda \cdot f)(x)=\lambda \times f(x) .
$$

Then $\mathcal{R}^{X}$ is a semi-module over $\mathcal{R}$.

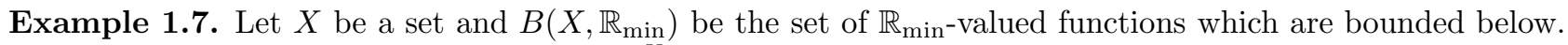
Then $B\left(X, \mathbb{R}_{\min }\right)$ is a sub-semi-module of $\left(\mathbb{R}_{\text {min }}^{X}, \min ,+\right)$.

Definition 1.8. Let $(\mathcal{R},+, \times)$ be a semi-ring, $(X,+, \cdot)$ and $(Y,+, \cdot)$ be two semi-modules over $\mathcal{R}$, and $L: X \rightarrow$ $Y$. One says that $L$ is a linear operator if $\forall \lambda, \mu \in \mathcal{R}, \forall x, y \in X, L(\lambda \cdot x+\mu \cdot y)=\lambda \cdot L(x)+\mu \cdot L(y)$.

Example 1.9. Let $X$ be a set and $K: X^{2} \rightarrow \mathbb{R}_{\min }$ be bounded below. Let $\mathcal{K}$ be the mapping from $B\left(X, \mathbb{R}_{\min }\right)$ to itself which maps $u$ to $\mathcal{K} u$ where

$$
\forall x \in X, \quad(\mathcal{K} u)(x)=\inf _{y \in X}\{K(x, y)+u(y)\} .
$$

Then $\mathcal{K}$ is a linear operator. 
Definition 1.10. Let $(\mathcal{R},+, \times)$ be a semi-ring, $(X,+, \cdot)$ be a semi-module over $\mathcal{R}, L: X \rightarrow X$ be a linear operator, and $\lambda \in \mathcal{R}$. One says that $\lambda$ is an eigenvalue of $L$ if there exists $x \in X$ such that $x \neq 0$ and $L(x)=\lambda \cdot x$. In this case, one says that $x$ is an eigenvector associated to $\lambda$.

Example 1.11. Same notations as example (1.9). Then $\lambda \in \mathbb{R}_{\min }$ is an eigenvalue of $\mathcal{K}$ if there exists $u \in$ $B\left(X, \mathbb{R}_{\text {min }}\right)$ such that $u \not \equiv+\infty$ and

$$
\forall x \in X, \quad \inf _{y \in X}\{K(x, y)+u(y)\}=\lambda+u(x) .
$$

\section{Spectral theory over $\mathbb{R}_{\text {min }}$}

Theorem 2.1. Let $X$ be a set and $K: X^{2} \rightarrow \mathbb{R}$ be bounded below. Suppose that there exists $\lambda \in \mathbb{R}$ and $u: X \rightarrow \mathbb{R}$ bounded below satisfying (1.1). Then

$$
\lambda=\inf _{\left(x_{n}\right) \in X^{\mathbb{N}}} \liminf _{n \rightarrow+\infty} \frac{K\left(x_{0}, x_{1}\right)+\cdots+K\left(x_{n-1}, x_{n}\right)}{n} .
$$

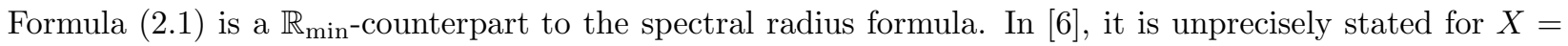
$[0,1]$. In [16], it is stated as here, but only for a finite set $X$ (in this case, formula (4.1) below is more interesting). For an interpretation of formula (2.1) in terms of spectral radius in a normed semi-algebra, one can refer to [3].

Proof. Let $\left(x_{n}\right) \in X^{\mathbb{N}}$. Then

$$
\forall n \in \mathbb{N}^{*}, \quad \lambda+u\left(x_{n-1}\right)=\inf _{y \in X}\left\{K\left(x_{n-1}, y\right)+u(y)\right\} \leq K\left(x_{n-1}, x_{n}\right)+u\left(x_{n}\right) .
$$

Adding the $n$ first equations, one gets

$$
\forall n \in \mathbb{N}^{*}, \quad n \lambda+u\left(x_{0}\right) \leq K\left(x_{0}, x_{1}\right)+\cdots+K\left(x_{n-1}, x_{n}\right)+u\left(x_{n}\right)
$$

Since $u$ is bounded, dividing by $n$ and passing to the limit $n \rightarrow+\infty$, one gets

$$
\lambda \leq \liminf _{n \rightarrow+\infty} \frac{K\left(x_{0}, x_{1}\right)+\cdots+K\left(x_{n-1}, x_{n}\right)}{n} .
$$

Since the sequence $\left(x_{n}\right)$ was arbitrary,

$$
\lambda \leq \inf _{\left(x_{n}\right) \in X^{\mathbb{N}}} \liminf _{n \rightarrow+\infty} \frac{K\left(x_{0}, x_{1}\right)+\cdots+K\left(x_{n-1}, x_{n}\right)}{n} .
$$

To prove the opposite inequality, let $\varepsilon>0$ and $y_{0} \in X$. One can construct inductively $\left(y_{n}\right) \in X^{\mathbb{N}}$ such that

$$
\forall n \in \mathbb{N}^{*}, \quad K\left(y_{n-1}, y_{n}\right)+u\left(y_{n}\right) \leq \inf _{x \in X}\left\{K\left(y_{n-1}, x\right)+u(x)\right\}+\varepsilon=\lambda+u\left(y_{n-1}\right)+\varepsilon
$$

Adding the $n$ first equations and dividing by $n$, one gets as in the first part of the proof,

$$
\forall n \in \mathbb{N}^{*}, \quad \frac{K\left(y_{0}, y_{1}\right)+\cdots+K\left(y_{n-1}, y_{n}\right)}{n}+\frac{u\left(y_{n}\right)}{n} \leq \lambda+\frac{u\left(y_{0}\right)}{n}+\varepsilon .
$$

So letting $n$ go to $+\infty$, one gets

$$
\lambda \geq \liminf _{n \rightarrow+\infty} \frac{K\left(y_{0}, y_{1}\right)+\cdots+K\left(y_{n-1}, y_{n}\right)}{n}-\varepsilon \geq \inf _{\left(x_{n}\right) \in X^{\mathbb{N}}} \liminf _{n \rightarrow+\infty} \frac{K\left(x_{0}, x_{1}\right)+\cdots+K\left(x_{n-1}, x_{n}\right)}{n}-\varepsilon .
$$


Theorem 2.2. Let $(X, d)$ be a compact metric space and $K \in \mathrm{C}^{0}\left(X^{2}, \mathbb{R}\right)$. Then there exists a unique $\lambda \in \mathbb{R}$ such that there exists $u \in \mathrm{C}^{0}(X, \mathbb{R})$ satisfying (1.1).

This is a $\mathbb{R}_{\min }$-counterpart to the Krein-Rutman Theorem. In [5], the proof is given for any finite set $X$. In [7], the proof is given for $X=[0,1]$, and it is noticed that "the proof method would hold for various abstractions". In [9], the proof is extended to $X=[0,1]^{n}$. In [14], a proof is given in the general setting with even weaker assumptions. But the proof method in [14] is somewhat different from that used in [7,9], and also less clear. The proof below is a direct generalization of the one used in $[7,9]$.

Proof. Set $E=\mathrm{C}^{0}(X, \mathbb{R})$. For all $u \in E$, set

$$
\|u\|=\sup _{x \in X}|u(x)|
$$

Then $E$ is a Banach space. For all $u \in E$ and $x \in X$, set

$$
(T u)(x)=\inf _{y \in X}\{K(x, y)+u(y)\}-\inf _{z \in X} \inf _{y \in X}\{K(z, y)+u(y)\} .
$$

The set $T(E)$ is equicontinuous. Indeed, let $\varepsilon>0$. Since $K$ is uniformly continuous, there exists $\alpha>0$ such that for all $x, y, x^{\prime}, y^{\prime} \in X$,

$$
\max \left\{d\left(x, x^{\prime}\right) ; d\left(y, y^{\prime}\right)\right\} \leq \alpha \Rightarrow\left|K(x, y)-K\left(x^{\prime}, y^{\prime}\right)\right| \leq \varepsilon .
$$

Let $x, x^{\prime} \in X$ be such that $d\left(x, x^{\prime}\right) \leq \alpha$. Then for all $u \in E$,

$$
\begin{aligned}
(T u)(x)-(T u)\left(x^{\prime}\right) & =\inf _{y \in X}\{K(x, y)+u(y)\}-\inf _{y \in X}\left\{K\left(x^{\prime}, y\right)+u(y)\right\} \\
& \leq \inf _{y \in X}\left\{K\left(x^{\prime}, y\right)+\varepsilon+u(y)\right\}-\inf _{y \in X}\left\{K\left(x^{\prime}, y\right)+u(y)\right\}=\varepsilon .
\end{aligned}
$$

Exchanging the roles of $x$ and $x^{\prime}$, one gets $\left|(T u)(x)-(T u)\left(x^{\prime}\right)\right| \leq \varepsilon$.

The function $T: E \rightarrow E$ which maps $u$ onto $T u$ is continuous. Indeed, let $u, v \in E$. For all $x \in X$,

$$
\begin{aligned}
(T v)(x) & =\inf _{y \in X}\{K(x, y)+v(y)-u(y)+u(y)\}-\inf _{z \in X} \inf _{y \in X}\{K(z, y)+v(y)-u(y)+u(y)\} \\
& \leq(T u)(x)+\sup _{y \in X}\{v(y)-u(y)\}-\inf _{y \in X}\{v(y)-u(y)\} \\
& \leq(T u)(x)+2\|v-u\| .
\end{aligned}
$$

Exchanging the role of $v$ and $u$, one gets $\|T v-T u\| \leq 2\|v-u\|$.

Now set

$$
K_{-}=\inf _{x, y \in X} K(x, y), \quad K_{+}=\sup _{x, y \in X} K(x, y), \quad C=\left\{u \in E ; \forall x \in X, 0 \leq u(x) \leq K_{+}-K_{-}\right\} .
$$

For all $u \in E$ and $x \in X$,

$$
0 \leq(T u)(x) \leq \inf _{y \in X}\left\{K_{+}+u(y)\right\}-\inf _{z \in X} \inf _{y \in X}\left\{K_{-}+u(y)\right\}=K_{+}-K_{-} .
$$

So $T(E) \subset C$. In particular, $T(C) \subset C$ and $T(C)$ is bounded. Since $T(E)$ is equicontinuous, $T(C)$ is equicontinuous too. According to the Ascoli-Arzela Theorem, $T(C)$ is relatively compact in $E$. Notice that $C$ is a closed convex subset of $E$. Recall Schauder Theorem: a continuous mapping from a closed convex subset $C$ of 
a Banach space into a compact subset of $C$ has a fixed point. So there is a $u \in C$ such that $T u=u$, which means that (1.1) is satisfied with

$$
\lambda=\inf _{z \in X} \inf _{y \in X}\{K(z, y)+u(y)\}
$$

The uniqueness of the eigenvalue $\lambda$ follows from Theorem 2.1.

\section{Parameter dependant problems}

Parameter dependent min-plus eigenvalue problems don't seem to have been studied in the min-plus literature.

Proposition 3.1. Let $(X, d)$ be a compact metric space, $\Omega$ be a topological space, and $K: \alpha \mapsto K_{\alpha}$ be a continuous function from $\Omega$ to $\mathrm{C}^{0}\left(X^{2}, \mathbb{R}\right)$ with the sup norm. For all $\alpha \in \Omega$, let $\lambda_{\alpha}$ be the unique real number associated to $K_{\alpha}$ by Theorem 2.2. Then the function $\alpha \longmapsto \lambda_{\alpha}$ from $\Omega$ to $\mathbb{R}$ is continuous.

Proof. Let $\alpha \in \Omega$ and $\varepsilon>0$. There is a neighborhood $\mathcal{V}$ of $\alpha$, such that

$$
\beta \in \mathcal{V} \Rightarrow \sup _{x, y \in X}\left|K_{\alpha}(x, y)-K_{\beta}(x, y)\right| \leq \varepsilon
$$

Then for all $\left(x_{n}\right) \in X^{\mathbb{N}}$ and $n \in \mathbb{N}^{*}$,

$$
\begin{aligned}
\frac{K_{\alpha}\left(x_{0}, x_{1}\right)+\cdots+K_{\alpha}\left(x_{n-1}, x_{n}\right)}{n}-\varepsilon & \leq \frac{K_{\beta}\left(x_{0}, x_{1}\right)+\cdots+K_{\beta}\left(x_{n-1}, x_{n}\right)}{n} \\
& \leq \frac{K_{\alpha}\left(x_{0}, x_{1}\right)+\cdots+K_{\alpha}\left(x_{n-1}, x_{n}\right)}{n}+\varepsilon .
\end{aligned}
$$

Taking first the liminf as $n \rightarrow+\infty$ in these inequalities, then taking the infimum over all $\left(x_{n}\right) \in X^{\mathbb{N}}$, and recalling formula $(2.1)$, one gets

$$
\lambda_{\alpha}-\varepsilon \leq \lambda_{\beta} \leq \lambda_{\alpha}+\varepsilon
$$

Proposition 3.2. Let $(X, d)$ be a compact metric space and $\Omega$ be a convex subset of a real vector space. For all $\alpha \in \Omega$, let $K_{\alpha} \in \mathrm{C}^{0}\left(X^{2}, \mathbb{R}\right)$. Suppose that for all $x, y \in X$, the function $\alpha \mapsto K_{\alpha}(x, y)$ form $\Omega$ to $\mathbb{R}$ is concave. For all $\alpha \in \Omega$, let $\lambda_{\alpha}$ be the unique real number associated to $K_{\alpha}$ by Theorem 2.2. Then the function $\alpha \mapsto \lambda_{\alpha}$ from $\Omega$ to $\mathbb{R}$ is concave.

In [6], this proposition is unprecisely stated for $X=[0,1]$ and $\Omega=\mathbb{R}$.

Proof. For all $\mathbf{x}=\left(x_{n}\right) \in X^{\mathbb{N}}, n \in \mathbb{N}^{*}$ and $\alpha \in \Omega$, set

$$
S(\mathbf{x}, n, \alpha)=\frac{K_{\alpha}\left(x_{0}, x_{1}\right)+\cdots+K_{\alpha}\left(x_{n-1}, x_{n}\right)}{n} .
$$

Let $t \in(0,1)$ and $\alpha, \beta \in \Omega$. Then for all $\mathbf{x} \in X^{\mathbb{N}}$ and $n \in \mathbb{N}^{*}$,

$$
S(\mathbf{x}, n, t \cdot \alpha+(1-t) \cdot \beta) \geq t S(\mathbf{x}, n, \alpha)+(1-t) S(\mathbf{x}, n, \beta)
$$

because of the concavity assumption. Because of the properties of the liminf, one gets for all $\mathbf{x} \in X^{\mathbb{N}}$

$$
\liminf _{n \rightarrow+\infty} S(\mathbf{x}, n, t \cdot \alpha+(1-t) \cdot \beta) \geq t \liminf _{n \rightarrow+\infty} S(\mathbf{x}, n, \alpha)+(1-t) \liminf _{n \rightarrow+\infty} S(\mathbf{x}, n, \beta)
$$


Taking the infimum over all $\mathbf{x} \in X^{\mathbb{N}}$, one gets

$$
\inf _{\mathbf{x} \in X^{\mathbb{N}}} \liminf _{n \rightarrow+\infty} S(\mathbf{x}, n, t \cdot \alpha+(1-t) \cdot \beta) \geq t \inf _{\mathbf{x} \in X^{\mathbb{N}}} \liminf _{n \rightarrow+\infty} S(\mathbf{x}, n, \alpha)+(1-t) \inf _{\mathbf{x} \in X^{\mathbb{N}}} \liminf _{n \rightarrow+\infty} S(\mathbf{x}, n, \beta) .
$$

So according to formula (2.1),

$$
\lambda_{t \cdot \alpha+(1-t) \cdot \beta} \geq t \lambda_{\alpha}+(1-t) \lambda_{\beta}
$$

\section{Numerical Methods}

The following proposition proves the convergence of the numerical method used in [6].

Proposition 4.1. Let $(X, d)$ be a compact metric space. Let $K: X^{2} \rightarrow \mathbb{R}$ be a lipschitz continuous function with lipschitz constant $\kappa: \forall x, x^{\prime}, y, y^{\prime} \in X,\left|K(x, y)-K\left(x^{\prime}, y^{\prime}\right)\right| \leq \kappa \max \left\{d\left(x, x^{\prime}\right) ; d\left(y, y^{\prime}\right)\right\}$. From Theorem 2.2, let $\lambda$ be the unique real number such that there exists $u \in \mathrm{C}^{0}(X, \mathbb{R})$ satisfying (1.1). Let $\left(X_{p}\right)_{p \in \mathbb{N}}$ be a sequence of finite subsets of $X$ such that

$$
h_{p}=\sup _{x \in X} \min _{y \in X_{p}} d(x, y) \underset{p \rightarrow+\infty}{\longrightarrow} 0 .
$$

From Theorem 2.2, for all $p \in \mathbb{N}$, let $\lambda_{p}$ be the unique real number such that there exists $u_{p} \in \mathbb{R}^{X_{p}}$ satisfying

$$
\forall x \in X_{p}, \quad \min _{y \in X_{p}}\left\{K(x, y)+u_{p}(y)\right\}=\lambda_{p}+u_{p}(x) .
$$

Then $\lambda \leq \lambda_{p} \leq \lambda+\kappa h_{p}$ and $\lambda_{p} \rightarrow \lambda$ as $p \rightarrow+\infty$.

The non-standard analysis point of view of [10], which considers "infinitely large" values of $p$, is related to the previous proposition.

Proof. Let $p \in \mathbb{N}$. From formula (2.1),

$$
\begin{aligned}
\lambda & =\inf _{\left(x_{n}\right) \in X^{\mathbb{N}}} \liminf _{n \rightarrow+\infty} \frac{K\left(x_{0}, x_{1}\right)+\cdots+K\left(x_{n-1}, x_{n}\right)}{n}, \\
\lambda_{p} & =\inf _{\left(x_{n}\right) \in X_{p}^{\mathbb{N}}} \liminf _{n \rightarrow+\infty} \frac{K\left(x_{0}, x_{1}\right)+\cdots+K\left(x_{n-1}, x_{n}\right)}{n} .
\end{aligned}
$$

On one side, $X_{p} \subset X$, so it is clear that $\lambda \leq \lambda_{p}$. On the other side, let $\varepsilon>0$. There exists $\left(x_{n}\right) \in X^{\mathbb{N}}$ such that

$$
\lambda \leq \liminf _{n \rightarrow+\infty} \frac{K\left(x_{0}, x_{1}\right)+\cdots+K\left(x_{n-1}, x_{n}\right)}{n} \leq \lambda+\varepsilon .
$$

By hypothesis, $\forall n \in \mathbb{N}, \exists y_{n} \in X_{p}, d\left(x_{n}, y_{n}\right) \leq h_{p}$. But $K$ is lipschitz-continuous, so $\forall n \in \mathbb{N}, \mid K\left(x_{n}, x_{n+1}\right)-$ $K\left(y_{n}, y_{n+1}\right) \mid \leq \kappa h_{p}$. In conclusion,

$$
\lambda_{p} \leq \liminf _{n \rightarrow+\infty} \frac{K\left(y_{0}, y_{1}\right)+\cdots+K\left(y_{n-1}, y_{n}\right)}{n} \leq \liminf _{n \rightarrow+\infty} \frac{K\left(x_{0}, x_{1}\right)+\cdots+K\left(x_{n-1}, x_{n}\right)}{n}+\kappa h_{p} \leq \lambda+\varepsilon+\kappa h_{p} .
$$

Since $\varepsilon$ was arbitrary, one gets $\lambda_{p} \leq \lambda+\kappa h_{p}$. 
Proposition 4.2. If $X_{p}$ has $q$ elements, then

$$
\lambda_{p}=\min _{1 \leq n \leq q} \min _{\left(x_{0}, \ldots, x_{n-1}\right) \in X_{p}^{n}} \frac{K\left(x_{0}, x_{1}\right)+\cdots+K\left(x_{n-1}, x_{0}\right)}{n} .
$$

Proof. Refer to [5] for example.

This formula shows that $\lambda_{p}$ is the "minimum cycle mean" and that it can be computed with a finite number of operations. The proof is similar to that of (2.1). Anyway, formula (4.2) isn't used in practice. There are better algorithms, such as Karp's which needs $O\left(q^{3}\right)$ operations, or Howard's which seems to be the fastest [8]. Notice that for the numerical analysis of min-plus eigenvalue problems, the matrices involved are full and very big (the bigger the better). So efficient algorithms are welcome, especially when the problem depends on a parameter which is varied such as in the next sections. Karp's algorithm is very easy to implement whereas Howard's algorithm is available through Scilab's Maxplus toolbox (see www-rocq.inria.fr/scilab/ and www-rocq.inria.fr/scilab/contributions.html).

\section{Periodic Kernels}

Proposition 5.1. Let $(X,+)$ be an abelian topological group, $K: X^{2} \rightarrow \mathbb{R}$ be bounded below, and $P$ be a subgroup of $X$. Suppose that

$$
\forall p \in P, \forall(x, y) \in X^{2}, K(x+p, y+p)=K(x, y)
$$

Let $(\mathbf{X},+)$ be the topological group which is the quotient of $X$ by $P$. For all $\mathbf{x}, \mathbf{y} \in \mathbf{X}$, let $x \in \mathbf{x}$ and set

$$
\mathbf{K}(\mathbf{x}, \mathbf{y})=\inf _{y \in \mathbf{y}} K(x, y)
$$

Let $\lambda \in \mathbb{R}$

- If $u: X \rightarrow \mathbb{R}$ is continuous and satisfies for all $p \in P$ and $x \in X, u(x+p)=u(x)$ and

$$
\inf _{y \in X}\{K(x, y)+u(y)\}=\lambda+u(x)
$$

then the quotient function $\mathbf{u}: \mathbf{X} \rightarrow \mathbb{R}$ deduced from $u$ is continuous and satisfies for all $\mathbf{x} \in \mathbf{X}$,

$$
\inf _{\mathbf{y} \in \mathbf{X}}\{\mathbf{K}(\mathbf{x}, \mathbf{y})+\mathbf{u}(\mathbf{y})\}=\lambda+\mathbf{u}(\mathbf{x})
$$

- Conversely, if $\mathbf{u}: \mathbf{X} \rightarrow \mathbb{R}$ is continuous and satisfies (5.3), then the P-periodic function $u: X \rightarrow \mathbb{R}$ deduced from $\mathbf{u}$ is continuous and satisfies (5.2).

This proposition is proved for $X=\mathbb{R}$ and $P=\mathbb{Z}$ in [6], and for $X=\mathbb{R}^{n}$ and $P=\mathbb{Z}^{n}$ in [9].

Proof. First notice that $\mathbf{K}$ is well defined since for all $x \in X, \mathbf{y} \in \mathbf{X}$ and $p \in P$,

$$
\inf _{y \in \mathbf{y}} K(x+p, y)=\inf _{y \in \mathbf{y}} K(x, y-p)=\inf _{y \in \mathbf{y}} K(x, y)
$$

The rest easily follows from the fact that if $\mathbf{x} \in \mathbf{X}$ and $x \in \mathbf{x}$, then

$$
\inf _{y \in X}\{K(x, y)+u(y)\}=\inf _{\mathbf{y} \in \mathbf{X}} \inf _{y \in \mathbf{y}}\{K(x, y)+u(y)\}=\inf _{\mathbf{y} \in \mathbf{X}} \inf _{y \in \mathbf{y}}\{K(x, y)+\mathbf{u}(\mathbf{y})\}=\inf _{\mathbf{y} \in \mathbf{X}}\{\mathbf{K}(\mathbf{x}, \mathbf{y})+\mathbf{u}(\mathbf{y})\}
$$




\section{ExAmples}

For all $\alpha \in \mathbb{R}$, let $K_{\alpha} \in \mathrm{C}^{0}\left(\mathbb{R}^{2}, \mathbb{R}\right)$. Suppose that for all $x, y \in \mathbb{R}$ and $\alpha \in \mathbb{R}$,

$$
\begin{aligned}
& K_{0}(x+1, y+1)=K_{0}(x, y), \\
& K_{\alpha}(x, y)=K_{0}(x, y)-\alpha(x-y) .
\end{aligned}
$$

For all $\alpha \in \mathbb{R}$, set

$$
\lambda_{\alpha}=\inf _{\left(x_{n}\right) \in \mathbb{R}^{\mathbb{N}}} \liminf _{n \rightarrow+\infty} \frac{K_{\alpha}\left(x_{0}, x_{1}\right)+\cdots+K_{\alpha}\left(x_{n-1}, x_{n}\right)}{n} .
$$

Since $\mathbb{R} / \mathbb{Z}$ is compact, it follows from Theorem 2.2 and Proposition 5.1 that $\lambda_{\alpha}$ is the unique real number such that there exists $u_{\alpha} \in \mathrm{C}^{0}(\mathbb{R}, \mathbb{R})$ periodic of period 1 satisfying

$$
\forall x \in \mathbb{R}, \inf _{y \in \mathbb{R}}\left\{K_{\alpha}(x, y)+u_{\alpha}(y)\right\}=\lambda_{\alpha}+u_{\alpha}(x)
$$

From Proposition 3.2, the function $\alpha \mapsto \lambda_{\alpha}$ is concave, so it has a right derivative $\frac{\mathrm{d} \lambda}{\mathrm{d} \alpha}\left(\alpha^{+}\right)$for all $\alpha$, and the function $\alpha \mapsto \frac{\mathrm{d} \lambda}{\mathrm{d} \alpha}\left(\alpha^{+}\right)$is decreasing.

\subsection{Frenkel-Kontorova models}

Let $L \in \mathrm{C}^{0}\left(\mathbb{R}^{2}, \mathbb{R}\right)$. Suppose that for all $x, y \in \mathbb{R}, L(x+1, y+1)=L(x, y)$. For all $\alpha \in \mathbb{R}$ and $x, y \in \mathbb{R}$, set

$$
K_{\alpha}(x, y)=L(x, y)-\alpha(x-y)
$$

Then $K_{\alpha}$ satisfies assumptions (6.1) and (6.2). A particular case of this situation arises when $V \in \mathrm{C}^{0}(\mathbb{R}, \mathbb{R})$ is periodic of period 1 , and for all $x, y \in \mathbb{R}$,

$$
L(x, y)=V(x)+\frac{(y-x)^{2}}{2}
$$

Figure 1 represents the dependence of $\lambda_{\alpha}$ with respect to $\alpha$ when $V(x)=C[1-\cos (2 \pi x)]$, where $C$ is another parameter. This example was introduced by Frenkel and Kontorova in 1938. The parameter $C$ is taken to be $(4 / 3) /(2 \pi)^{2}$. The set $\left\{0, \frac{1}{p}, \frac{2}{p}, \ldots, \frac{p-1}{p}\right\}$ was used as a discretization of $\mathbb{R} / \mathbb{Z}$. Notice that for $\alpha \in\left[0, \frac{1}{2}\right]$ and $x, y \in[0,1]$,

$$
\inf _{p \in \mathbb{Z}} K_{\alpha}(x, y+p)=V(x)+\inf _{p \in\{-1,0,1\}}\left\{\frac{(y-x+p)^{2}}{2}-\alpha(x-y-p)\right\}
$$

Plot (a) illustrates the continuity of $\alpha \mapsto \lambda_{\alpha}$. Plot (b) suggests that $\frac{\mathrm{d} \lambda}{\mathrm{d} \alpha}\left(\alpha^{+}\right)$may also be a continuous function of $\alpha$, but like a devil's-staircase. It is not clear whether this can be deduced from the results of Aubry $[1,2]$ and from Griffiths' remarks in [13].

\subsection{Homogenization of Hamilton-Jacobi equations}

Let $L \in \mathrm{C}^{0}\left(\mathbb{R}^{2}, \mathbb{R}\right)$. Suppose that for all $x, v \in \mathbb{R}, L(x+1, v)=L(x, v)$. For all $\alpha \in \mathbb{R}$ and $x, y \in \mathbb{R}$, set

$$
K_{\alpha}(x, y)=\inf \left\{\int_{0}^{1} L(\xi(s), \dot{\xi}(s)) \mathrm{d} s ; \xi \in \mathrm{C}^{1}([0,1], \mathbb{R}), \xi(0)=y, \xi(1)=x\right\}-\alpha(x-y)
$$




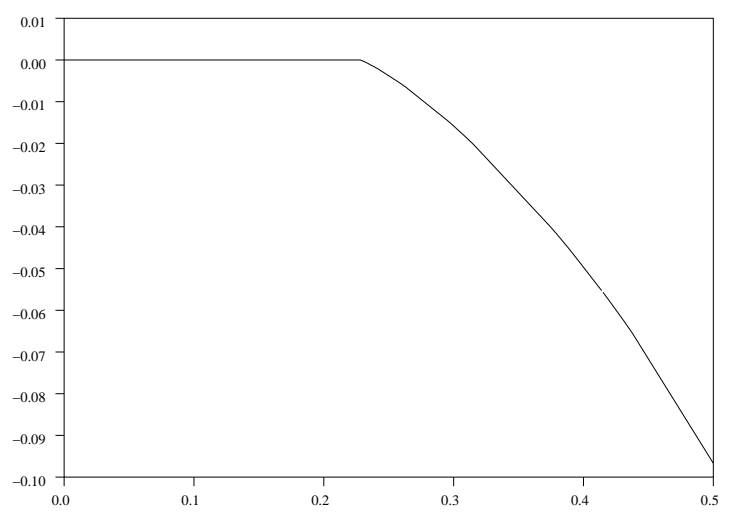

(a) $\alpha \mapsto \lambda_{\alpha}$

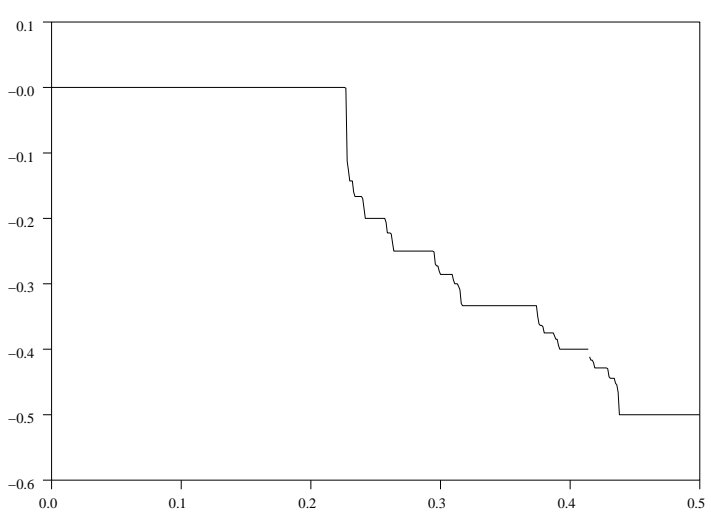

(b) $\alpha \mapsto \frac{\mathrm{d} \lambda}{\mathrm{d} \alpha}$

Figure 1. The minimum energy in the Frenkel-Kontorova model.

Then $K_{\alpha}$ satisfies assumptions (6.1) and (6.2). A particular case of this situation arises when $V \in \mathrm{C}^{0}(\mathbb{R}, \mathbb{R})$ is periodic of period 1 , and for all $x, v \in \mathbb{R}$,

$$
L(x, v)=V(x)+\frac{v^{2}}{2}
$$

For this situation, there is an almost explicit formula for the eigenvalue $\lambda_{\alpha}$ (see [9] for example), namely

$$
\lambda_{\alpha}= \begin{cases}\min V & \text { if }|\alpha| \leq \int_{0}^{1} \sqrt{2[V(x)-\min V]} \mathrm{d} x \\ \lambda \operatorname{such} \text { that }|\alpha|=\int_{0}^{1} \sqrt{2[V(x)-\lambda]} \mathrm{d} x & \text { if }|\alpha|>\int_{0}^{1} \sqrt{2[V(x)-\min V]} \mathrm{d} x .\end{cases}
$$

Figure 2 represents the dependance of $\lambda_{\alpha}$ with respect to $\alpha$ when $V(x)=1-\cos (2 \pi x)$. Notice the similarities and differences with Figure 1. It seems strange that these two closely related models exhibit such different behaviors.

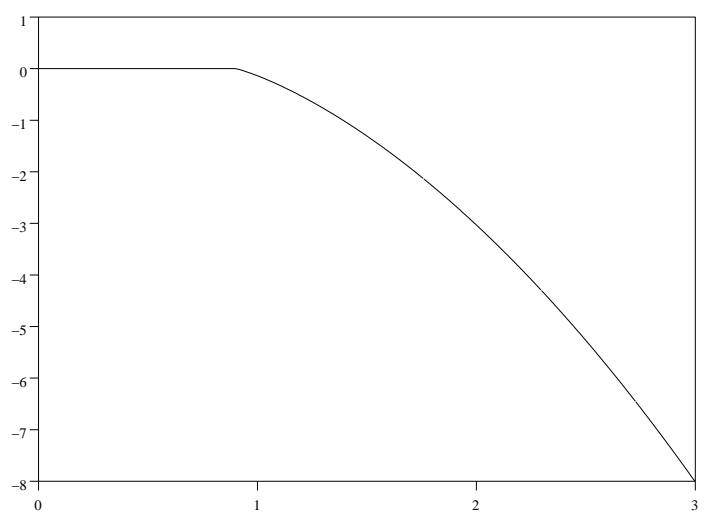

(a) $\alpha \mapsto \lambda_{\alpha}$

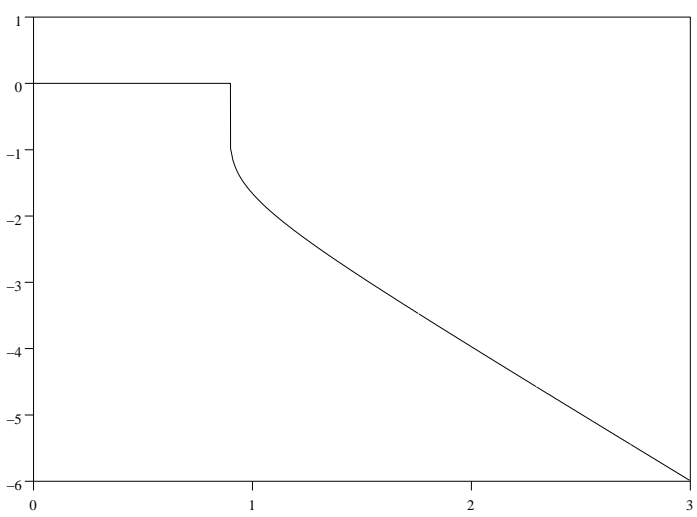

(b) $\alpha \mapsto \frac{\mathrm{d} \lambda}{\mathrm{d} \alpha}$

FigurE 2. The effective hamiltonian for the eikonal equation. 
When $L(x, v)$ is convex with respect to $v$, it is proved in [9] that the eigenvalue problem (6.4) with kernel $K_{\alpha}$ given by $(6.5)$ is equivalent to the cell-problem

$$
H\left(x, \alpha+\frac{\partial u}{\partial x}(x)\right)=\bar{H}(\alpha)
$$

where $\bar{H}(\alpha)=-\lambda_{\alpha}$ and for all $x, p \in \mathbb{R}$,

$$
H(x, p)=\sup _{v \in \mathbb{R}}\{p \cdot v-L(x, v)\}
$$

Recall that this cell problem comes from the homogenization as $\varepsilon$ tends to 0 of the equation

$$
\frac{\partial v}{\partial t}(t, x)+H\left(\frac{x}{\varepsilon}, \frac{\partial v}{\partial x}(t, x)\right)=0
$$

Other links between Aubry-Mather theory and Hamilton-Jacobi equations can be found in [11]. Notice also that the numerical method which is rather straightforward for the Frenkel-Kontorova model doesn't seem very good for Hamilton-Jacobi equations because the kernel $K_{\alpha}(x, y)$ given by $(6.5)$ is already diificult to compute; In some cases, one could use a software which solves the two point boundary value problem arising from the Euler-Lagrange equation associated to (6.5). This needs further investigation.

\subsection{On the convergence rate}

Let us return to Frenkel-Kontorova models. Following [6], let $V: \mathbb{R} \rightarrow \mathbb{R}$ be the 1-periodic piecewise parabolic function defined by

$$
V(x)= \begin{cases}\frac{c}{2} x^{2} & \text { if }-\frac{1}{4} \leq x \leq \frac{1}{4} \\ \frac{c}{16}-\frac{c}{2}\left(x-\frac{1}{2}\right)^{2} & \text { if } \frac{1}{4} \leq x \leq \frac{3}{4}\end{cases}
$$

with $c \geq 0$. The set $\left\{0, \frac{1}{p}, \frac{2}{p}, \ldots, \frac{p-1}{p}\right\}$ is used as a discretization. According to Proposition 4.1 with $h_{p}=\frac{1}{p}$, the following inequality is true:

$$
\log _{10}\left(\lambda_{p}-\lambda\right) \leq \log _{10}(\kappa)-\log _{10}(p)
$$

where $\kappa$ is the lipschitz constant of $\mathbf{K}$ defined by (5.1). Suppose $c=\frac{4}{3}$ and $\alpha=\frac{13}{32}$. Then $\lambda$ can be computed explicitly as indicated in [6], namely $\lambda=-\frac{265}{2048}$. Figure 3 plots $\log _{10}\left(\lambda_{p}-\lambda\right)$ as a function of $-\log _{10}(p)$. In fact, due to special properties of $K$, the slope of the function $\log _{10}\left(\lambda_{p}-\lambda\right)$ seems to be close to 2 , which suggests that the error is quadratic. However, one can construct some artificial examples of minplus eigenvalue problems in which the convergence rate is only linear. Precise assumptions on the kernel for the convergence to be quadratic remain to be found. viscosity methods in pde's, and also Lin Yi-Jiun for support. 


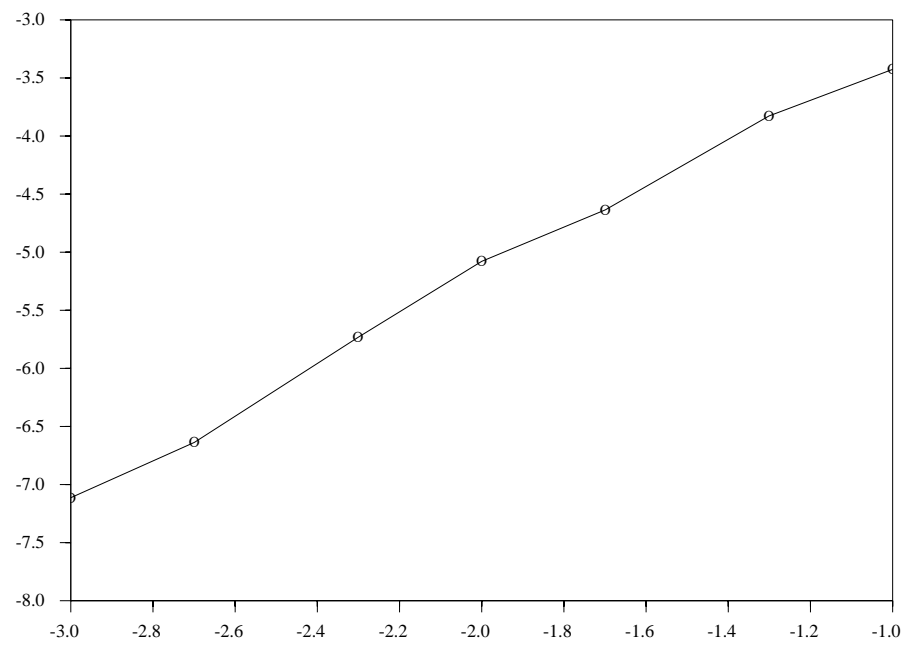

Figure 3. Convergence. $\log _{10}\left(\lambda_{p}-\lambda\right)$ versus $-\log _{10}(p)$.

\section{REFERENCES}

[1] S. Aubry, The new concept of transitions by breaking of analyticity in a crystallographic model, in Solitons and Condensed Matter Physics, A.R. Bishop and T. Schneider, Eds., Springer-Verlag, Berlin (1978) 264-277.

[2] S. Aubry, The twist map, the extended Frenkel-Kontorova model and the devil's staircase. Physica D 7 (1983) $240-258$.

[3] N. Bacaër, Min-plus spectral theory and travelling fronts in combustion, in Proceedings of the Workshop on Max-Plus Algebras, Prague, August (2001). Submitted to S. Gaubert, Ed., Elsevier Science, Amsterdam.

[4] N. Bacaër, Can one use Scilab's max-plus toolbox to solve eikonal equations?, in Proceedings of the Workshop on Max-Plus Algebras, Prague, August (2001). Submitted to S. Gaubert, Ed., Elsevier Science, Amsterdam.

[5] F. Baccelli, G.J. Olsder, J.P. Quadrat and G. Cohen, Synchronization and Linearity. Wiley, Chichester (1992).

[6] W. Chou and R.B. Griffiths, Ground states of one-dimensional systems using effective potentials. Phys. Rev. B 34 (1986) 6219-6234.

[7] W. Chou and R.J. Duffin, An additive eigenvalue problem of physics related to linear programming. Adv. in Appl. Math. 8 (1987) 486-498.

[8] J. Cochet-Terrasson, G. Cohen, S. Gaubert, M. Mc Gettrick and J.P. Quadrat, Numerical computation of spectral elements in max-plus algebra. http://amadeus.inria.fr/gaubert/HOWARD.html

[9] M. Concordel, Periodic homogenization of Hamilton-Jacobi equations: additive eigenvalues and variational formula. Indiana Univ. Math. J. 45 (1996) 1095-1117.

[10] P.I. Dudnikov and S.N. Samborskii, Endomorphisms of semimodules over semirings with idempotent operation. Math. USSRIzv. 38 (1992) 91-105.

[11] L.C. Evans and D. Gomes, Effective Hamiltonians and averaging for Hamiltonian dynamics I. Arch. Rational Mech. Anal. 157 (2001) 1-33.

[12] J.S. Golan, The Theory of Semirings with Applications in Mathematics and Theoretical Computer Science. Longman Scientific \& Technical, Harlow (1992).

[13] R.B. Griffiths, Frenkel-Kontorova models of commensurate-incommensurate phase transitions, in Fundamental Problems in Statistical Mechanics. VII, H. van Beijeren, Ed., North-Holland, Amsterdam (1990) 69-110.

[14] V.N. Kolokoltsov and V.P. Maslov, Idempotent Analysis and its Applications. Kluwer Academic Publishers, Dordrecht, The Netherlands (1997).

[15] G. Namah and J.M. Roquejoffre, The "hump" effect in solid propellant combustion. Interfaces Free Bound 2 (2000) $449-467$.

[16] S.J. Sheu and A.D. Wentzell, On the solutions of the equation arising from the singular limit of some eigen problems, in Stochastic Analysis, Control, Optimization and Applications, W.M. McEneaney et al., Eds., Birkhäuser, Boston (1999) 135150. 